\title{
Tratamento de sementes com fungicidas e inseticidas como redutores dos efeitos do estresse hídrico em plantas de soja
}

\author{
Seed treatment with fungicides and insecticides reducing the hydric stress on soybean plants
}

\section{Ricardo Silveiro Balardin ${ }^{I^{*}}$ Felipe Dalla Lana da Silva ${ }^{\mathrm{I}}$ Daniel Debona $^{\mathrm{II}}$ Gerson Dalla Corte $^{\mathrm{I}}$ Diego Dalla Favera ${ }^{\mathrm{I}}$ Nédio Rodrigo Tormen ${ }^{\mathrm{I}}$}

\section{RESUMO}

O objetivo do trabalho foi avaliar o efeito de quatro tratamentos de sementes: água (T1), carbendazim + thiram (T2), fipronil + tiofanato metílico + piraclostrobina (T3) $e$ abamectina + thiamethoxam + fludioxonil + mefenoxan + thiabendazole (T4) sobre parâmetros de crescimento de plantas de soja submetidas a dois regimes hídricos (presença e ausência de estresse hídrico) estabelecidos logo após a emergência das plântulas. $O$ experimento foi conduzido em casa de vegetação e campo. Na casa de vegetação, nos tratamentos sem estresse hídrico, foram feitas quatro irrigações por dia e, nos tratamentos com estresse hídrico, foi realizada uma irrigação a cada três dias. Neste ensaio, foi avaliada a estatura de plantas, comprimento radicular, volume radicular, massa seca da parte aérea, massa seca radicular e área foliar. No ensaio de campo, o estresse hídrico foi estabelecido pela construção de túneis baixos de polietileno que impediram o fornecimento de água pela irrigação e chuva. No campo, foram avaliados estatura de planta, teor relativo de clorofila, emergência, ataque de lagarta-elasmo e rendimento de grãos. Nos dois ambientes, as plantas foram mantidas sob estresse até 28 dias após a emergência. Em casa de vegetação, todos os parâmetros foram influenciados pelo tratamento de sementes na presença e ausência de estresse hídrico. No campo, o tratamento com fipronil + tiofanato metílico + piraclostrobina (T3) $e$ abamectina + thiamethoxam + fludioxonil + mefenoxan + thiabendazole (T4) proporcionaram maior estatura de planta e teor relativo de clorofila em ambos os regimes hídricos e maior emergência e rendimento de grãos em plantas sem e com restrição hídrica, respectivamente. Conclui-se que o tratamento de sementes com esses produtos promove alterações benéficas nas plantas, aumentando sua tolerância ao estresse hídrico, com efeito positivo sobre o rendimento de grãos da soja.

Palavras-chave: Glycine max, efeito fisiológico, déficit hídrico, piraclostrobina, thiamethoxam.

\section{ABSTRACT}

The objective of this research was evaluate four seed treatments: water (T1), carbendazim + thiram (T2), fipronil + thiophanate methyl + pyraclostrobin (T3) and abamectin + thiamethoxam + fludioxonil + mefenoxan + thiabendazole (T4) on growth parameters of soybean plants with or without low water availability. The experiments were conducted under field conditions and greenhouse. In greenhouse, in treatments without low water availability, it was made four irrigations by day and in treatments with low water availability, it was made one irrigation each three days. In this trial, it was evaluated the plant height, root length, root volume, dry shoot weight, dry root weight and foliar area. In the field trial, low water availability was established by construction of low tunnels of polyethylene which prevented the water supply by irrigation and rain. In the field, it was also evaluated the plant height, the relative chlorophyll content, emergence, lesser cornstalk borer attack and grain yield. In the both environment, the plants were kept on stress until $28^{\text {th }}$ days after emergence. In greenhouse, all the parameters were influenced by seed treatments with or without low water availability. In field, the treatment with fipronil + thiophanate methyl + pyraclostrobin (T3) and abamectin + thiamethoxam + fludioxonil + mefenoxan + thiabendazole (T4) promoted higher plant height and relative chlorophyll content in both hydric schemes, higher emergence and grain yield in plants without and with lower water availability, respectively. It was concluded that seed treatment with these products promote benefit changes in plant, increasing its tolerance to hydric stress, with positive effect on soybean grain yield.

Key words: Glycine max, physiological effect, low water availability, pyraclostrobin, thiamethoxam.

'Departamento de Defesa Fitossanitária, Universidade Federal de Santa Maria (UFSM), Av. Roraima, 1000, 97105-900, Santa

Maria, RS,Brasil. E-mail: balardin@balardin.com. *Autor para correspondência.

IIDepartamento de Fitopatologia, Universidade Federal de Viçosa (UFV), Viçosa, MG, Brasil. 


\section{INTRODUÇÃO}

Muitos patógenos presentes no solo ou transmitidos via semente reduzem o estande de plantas de soja (COSTAMILAN et al., 2010). Dessa forma, o tratamento de sementes com fungicidas é uma alternativa barata e viável para a redução do dano causado por patógenos de semente. Pragas de solo também podem causar falhas na lavoura, pelo fato de se alimentarem de sementes, raízes e da parte aérea das plantas (BAUDET \& PESKE, 2007). Para a maioria dessas pragas, o tratamento de sementes também desempenha um papel primário na proteção das sementes e das plântulas, reduzindo o dano causado por elas.

O déficit hídrico é o mais importante e frequente estresse que afeta a cultura da soja. Em algumas regiões do Brasil, é comum a distribuição irregular das chuvas logo após a semeadura, acarretando redução no estande e no crescimento das plantas. O tratamento de sementes, além de promover o controle de patógenos e pragas, pode favorecer a emergência e o desenvolvimento de plantas submetidas a estresse hídrico. PEREIRA et al. (1993), trabalhando com diferentes períodos de seca na semeadura da soja, observaram que a emergência foi maior quando as sementes foram tratadas com o fungicida thiram do que na testemunha. Discos foliares de trigo tratados com estrobilurina (kresoxim-methyl) apresentaram maiores níveis de ácido abscísico, promovendo redução na abertura estomatal e no consumo de água (GROSSMANN et al., 1999).

Embora alguns estudos tenham demonstrado que o tratamento de sementes aumenta a emergência de plântulas e beneficia o crescimento das plantas submetidas a estresse hídrico, poucos trabalhos têm avaliado o efeito dessa prática na produtividade. Além disso, não há trabalhos que tenham avaliado o efeito da aplicação de estrobilurinas nas sementes e o crescimento de plantas sob condições de estresse hídrico. Portanto, o presente estudo teve como objetivo avaliar o efeito de quatro tratamentos de sementes sobre parâmetros de crescimento de plantas de soja submetidas a diferentes condições hídricas.

\section{MATERIAL E MÉTODOS}

Os experimentos foram desenvolvidos em casa de vegetação com controle parcial de temperatura $\left(18-30^{\circ} \mathrm{C}\right)$ e umidade $(75-100 \%)$, e em campo. Ambos situados no município de Itaara - RS. Os trabalhos foram realizados entre janeiro e maio de 2009.
O delineamento foi constituído de um fatorial 2x4. O fator A foi representado por dois regimes hídricos: ausência e presença de estresse hídrico. O fator B foi constituído de três tratamentos de semente e um tratamento com água: T1 - testemunha com a aplicação de água, T2 - carbendazim $(30,0 \mathrm{~g}$ de i.a. $100 \mathrm{~kg}$ de sementes $\left.^{-1}\right)+$ thiram $\left(70,0 \mathrm{~g}\right.$ de i.a. $100 \mathrm{~kg}$ de sementes $\left.{ }^{-1}\right)$, $\mathrm{T} 3$ - fipronil $\left(50,0 \mathrm{~g}\right.$ de i.a.100kg de sementes $\left.{ }^{-1}\right)+$ tiofanato metílico $\left(5,0 \mathrm{~g}\right.$ de i.a. $100 \mathrm{~kg}$ de sementes $\left.{ }^{-1}\right)+$ piraclostrobina (45,0g de i.a. $100 \mathrm{~kg}$ de sementes $\left.{ }^{-1}\right)$ e T4 - abamectina $\left(62,5 \mathrm{~g}\right.$ de i.a.100kg de sementes $\left.{ }^{-1}\right)+$ thiamethoxam $\left(70,0 \mathrm{~g}\right.$ de i.a. $100 \mathrm{~kg}$ de sementes $\left.{ }^{-1}\right)+$ fludioxonil $\left(2,5 \mathrm{~g}\right.$ de i.a. $100 \mathrm{~kg}$ de sementes $\left.{ }^{-1}\right)+$ mefenoxan $\left(1,0 \mathrm{~g}\right.$ de i.a. $100 \mathrm{~kg}$ de sementes $\left.{ }^{-1}\right)+$ thiabendazole $\left(15,0 \mathrm{~g}\right.$ de i.a. $100 \mathrm{~kg}$ de sementes $\left.{ }^{-1}\right)$. Para cada tratamento, uma calda de $6 \mathrm{~mL}$ (produto dosado+água) foi aplicada em $1 \mathrm{~kg}$ de semente.

Em casa de vegetação, os dois regimes hídricos foram obtidos pelo manejo da irrigação, utilizando um sistema de gotejamento tipo "espaguete". O solo foi mantido próximo da capacidade de campo em ambos os regimes hídricos até a emergência das plântulas. Após esse período, nas plantas não submetidas ao estresse hídrico, o sistema de irrigação foi regulado para fornecer água às plantas por 10 minutos quatro vezes ao dia. Nos tratamentos com estresse hídrico, a irrigação foi reduzida para 10 minutos a cada três dias. Aos 28 dias após a emergência, todos os tratamentos foram submetidos ao mesmo regime hídrico dos tratamentos sem estresse. O período de estresse hídrico foi definido em ensaio realizado previamente com objetivo de causar uma deficiência hídrica nas plantas sem que elas atingissem o ponto de murcha permanente.

No campo, quatro dias após a semeadura, foi realizada irrigação por aspersão com uma lâmina de $10 \mathrm{~mm}$ em todas as unidades experimentais. Sobre os tratamentos com estresse hídrico foram construídos túneis baixos cobertos com polietileno, os quais foram fechados antes de precipitações e à noite, com o objetivo de submeter as plantas ao estresse hídrico. Após o 28 dia de emergência das plantas, os túneis baixos foram removidos e todas as parcelas estiveram sujeitas a mesma condição hídrica, proveniente da chuva. No período em que os tratamentos com estresse hídrico permaneceram protegidos da chuva e irrigação, contabilizaram-se precipitações de $54(12 \mathrm{~mm}$ proveniente de irrigação), 57,25 e $15 \mathrm{~mm}$, na primeira, segunda, terceira e quarta semanas após a emergência, respectivamente.

Os ensaios foram conduzidos utilizando as cultivares de soja 'FUNDACEP 53 RR', em casa de vegetação e 'A 8100 RG', para a condição de campo. 
No ensaio em casa de vegetação, foram utilizados vasos plásticos de $5 \mathrm{~L}$, contendo substrato composto por areia e solo (3:1). O substrato continha as seguintes características químicas: $\mathrm{pH}_{\text {agua }}=5,3 ; \mathrm{Ca}=1,3 \mathrm{cmol}_{\mathrm{c}}$ $\mathrm{dm}^{-3} ; \mathrm{Al}=0,1 \mathrm{cmol}_{\mathrm{c}} \mathrm{dm}^{-3} ; \mathrm{P}_{\text {Mehlich }}=14,4 \mathrm{mg} \mathrm{dm}^{-3} ; \mathrm{K}=$ $36,0 \mathrm{mg} \mathrm{dm}^{-3}$ e $\mathrm{MO}=0,3 \%$. A fertilidade do solo foi corrigida segundo as recomendações da COMISSÃO DE QUÍMICAE FERTILIDADE DO SOLO (2004). Esse substrato corrigido foi misturado com casca de arroz, na proporção substrato e casca de arroz 3:2. Em cada vaso, foram semeadas dez sementes e, posteriormente, a população final foi ajustada para três plântulas por vaso

Os parâmetros avaliados foram estatura de planta, comprimento radicular, massa seca de raiz, massa seca da parte aérea e área foliar. Para avaliação da área foliar, todas as folhas de cada planta foram destacadas e colocadas sobre pano de fundo de coloração branca. Em seguida, foram obtidas imagens fotográficas, que foram usadas na estimativa da área foliar através do software QUANT $^{\oplus}$ (VALE et al., 2001), utilizando um pallete de 32 cores.

No ensaio de campo, a semeadura foi realizada manualmente em dois sulcos, com profundidade de três centímetros no dia 12 de janeiro de 2009. A densidade de semeadura utilizada foi de 322 mil sementes ha ${ }^{-1}$. As dimensões dos canteiros eram de $0,25 \times 1,5 \times 56 \mathrm{~m}$, com espaçamento de $0,5 \mathrm{~m}$ entre sulcos. Os tratamentos foram delineados em blocos ao acaso com quatro repetições. Cada unidade experimental consistiu em duas linhas de semeadura por sete metros de comprimento. Foram realizadas duas aplicações $\left(\mathrm{R}_{3}\right.$ e $\mathrm{R}_{5}$ ) dos fungicidas tebuconazol (200g i.a $\left.\mathrm{L}^{-1}\right)+$ carbendazim (500g i.a L ${ }^{-1}$ ), na dose de 0,50L de p.c ha ${ }^{-1}$.

Foi avaliada a porcentagem de emergência aos 7, 14, 21 e 28 dias após a emergência, a partir da contagem das plantas das duas linhas nos cinco metros centrais de cada parcela. A estatura de planta foi avaliada aos 7, 14, 28 e 42 dias após a emegência, em dez plantas em sequência de uma linha de semeadura no centro de cada parcela. $\mathrm{O}$ ataque de lagarta-elasmo (E. lignosellus) foi avaliado aos 35 dias após a emergência. Aos 28, 35 e 42 dias após a emergência, foi avaliado o teor relativo de clorofila com a utilização de clorofilômetro SPAD 502 (Minolta). Foram realizadas duas leituras em cada folíolo da penúltima folha totalmente expandida. Ao atingir a maturação, as plantas foram cortadas manualmente e trilhadas em batedor estacionário e as amostras foram pesadas. O rendimento de grãos foi convertido para $13 \%$ de umidade e expresso em $\mathrm{kg} \mathrm{ha}^{-1}$.

O nível de significância dos tratamentos foi determinado através da análise de variância (teste $\mathrm{F}$ a
$5 \%$ de probabilidade de erro). Quando os efeitos foram significativos, as médias foram comparadas pelo teste de Tukey $(\mathrm{P} \leq 0,05)$.

\section{RESULTADOS E DISCUSSÃO}

Ensaio de casa de vegetação

A análise de variância mostrou interação significativa entre os fatores regime hídrico e tratamento de sementes para todas as variáveis avaliadas $(\mathrm{P} \leq 0,05)$. As variáveis foram reduzidas pelo estresse hídrico (Tabela 1). Os tratamentos apresentaram diferenças significativas em função do regime hídrico. Na ausência de déficit hídrico, os tratamentos com fipronil + tiofanato metílico + piraclostrobina (T3) e abamectina + thiamethoxam + fludioxonil + mefenoxan + thiabendazole (T4) proporcionaram aumento na estatura de planta, volume radicular, massa seca radicular e área foliar em relação à testemunha, sem diferirem entre si. Somente os tratmentos com carbendazim + thiram (T2) e abamectina + thiamethoxam + fludioxonil + mefenoxan + thiabendazole (T4) promoveram aumento significativo na massa seca da parte aérea. $\mathrm{O}$ comprimento radicular não foi influenciado pelos tratamentos. Na presença de déficit hídrico, o tratamento com fipronil + tiofanato metílico + piraclostrobina (T3) proporcionou incremento significativo na estatura de planta, comprimento radicular, volume radicular, massa seca da parte aérea massa seca radicular e área foliar. Somente para os dados de estatura de planta e área foliar houve diferença entre os tratamentos com fipronil + tiofanato metílico + piraclostrobina (T3) e abamectina + thiamethoxam + fludioxonil + mefenoxan + thiabendazole (T4), sendo este inferior ao primeiro.

Dos compostos testados neste ensaio, foram encontrados relatos de efeitos fisiológicos via tratamento de sementes devido ao thiamethoxam (TAVARES et al., 2007; CASTRO et al., 2008) e das estrobilurinas aplicadas via foliar (GROSSMANN \& RETZLAFF, 1997; GROSSMANN et al., 1999; NASON et al., 2007). Da mesma forma que o encontrado no presente experimento, TAVARES et al. (2007) mostraram aumento na área foliar de plantas de soja cujas sementes foram tratadas com thiamethoxam. Em contrapartida, CASTRO et al. (2008) não encontraram efeito no crescimento de plantas de soja, porém verificaram que o raio médio radicular foi menor, o que pode aumentar a absorção de água e nutrientes.

O déficit hídrico reduz a taxa fotossintética das plantas, principalmente por induzir o fechamento estomático (SANTOS et al., 2004, 2006) e diminuir a expansão foliar (TAIZ \& ZEIGER, 2009), limitando a assimilação de $\mathrm{CO}_{2}$ (CORNIC, 2000), o que prejudica a 
Tabela 1 - Efeito de tratamentos de sementes na estatura $(\mathrm{EP})(\mathrm{cm})$, comprimento radicular $(\mathrm{CR})(\mathrm{cm})$, volume radicular $(\mathrm{VR})\left(\mathrm{cm}^{3}\right)$, massa seca de parte aérea (MSPA) (g), massa seca radicular (MSR) (g) e área foliar (AF) $\left(\mathrm{cm}^{2}\right.$ planta $\left.^{-1}\right)$ de plantas de soja da cultivar 'FUNDACEP 53 RR' na ausência e presença de estresse hídrico. Santa Maria, 2010.

\begin{tabular}{|c|c|c|c|c|c|c|}
\hline \multirow{2}{*}{ Tratamentos ${ }^{1}$} & \multicolumn{2}{|c|}{ 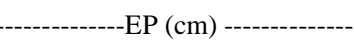 } & \multicolumn{2}{|c|}{ - } & \multicolumn{2}{|c|}{ - } \\
\hline & S.E. ${ }^{3}$ & C.E. ${ }^{4}$ & S.E. & C.E. & S.E. & C.E. \\
\hline $\mathrm{T} 1$ & $19,04 \mathrm{a}^{2}$ & $15,75 \mathrm{ab}$ & $34,42 \mathrm{a}$ & $26,02 \mathrm{a}$ & $11,00 \mathrm{a}$ & $4,38 \mathrm{a}$ \\
\hline $\mathrm{T} 2$ & $20,33 \mathrm{ab}$ & $15,87 \mathrm{ab}$ & $35,00 \mathrm{a}$ & $30,13 \mathrm{~b}$ & $11,25 \mathrm{a}$ & $6,75 \mathrm{~b}$ \\
\hline $\mathrm{T} 3$ & $22,33 \mathrm{c}$ & $17,92 \mathrm{~b}$ & $37,08 \mathrm{a}$ & $33,67 \mathrm{bc}$ & $14,25 \mathrm{~b}$ & $11,25 \mathrm{c}$ \\
\hline $\mathrm{T} 4$ & $21,64 \mathrm{bc}$ & 15,16 a & 37,17 a & $36,29 \mathrm{c}$ & $15,00 \mathrm{~b}$ & $11,50 \mathrm{c}$ \\
\hline Média & $20,83 \mathrm{~B}^{2}$ & $16,17 \mathrm{~A}$ & $35,91 \mathrm{~B}$ & $31,52 \mathrm{~A}$ & $12,87 \mathrm{~B}$ & $8,47 \mathrm{~A}$ \\
\hline $\mathrm{CV}(\%)$ & \multicolumn{2}{|c|}{5,64} & \multicolumn{2}{|c|}{5,41} & \multicolumn{2}{|c|}{10,16} \\
\hline \multirow{2}{*}{ Tratamentos ${ }^{1}$} & \multicolumn{2}{|c|}{ MSPA (g) - } & \multicolumn{2}{|c|}{ MSR (g) - } & \multicolumn{2}{|c|}{ AF $\left(\mathrm{cm}^{2}\right.$ planta $\left.^{-1}\right)$} \\
\hline & S.E. ${ }^{3}$ & C.E. ${ }^{4}$ & S.E. & C.E. & S.E. & C.E. \\
\hline $\mathrm{T} 1$ & $1,88 \mathrm{a}^{2}$ & $0,80 \mathrm{a}$ & $0,97 \mathrm{a}$ & $0,62 \mathrm{a}$ & $273,24 \mathrm{a}$ & $92,46 \mathrm{a}$ \\
\hline $\mathrm{T} 2$ & $2,18 \mathrm{~b}$ & $1,14 \mathrm{~b}$ & $1,05 \mathrm{ab}$ & $0,70 \mathrm{a}$ & $314,41 \mathrm{a}$ & $126,28 \mathrm{~b}$ \\
\hline $\mathrm{T} 3$ & $2,12 \mathrm{ab}$ & $1,56 \mathrm{c}$ & $1,18 \mathrm{bc}$ & $1,26 \mathrm{~b}$ & $378,65 \mathrm{~b}$ & $195,06 \mathrm{~d}$ \\
\hline $\mathrm{T} 4$ & $2,22 \mathrm{~b}$ & $1,44 \mathrm{c}$ & $1,30 \mathrm{c}$ & $1,20 \mathrm{~b}$ & $377,88 \mathrm{~b}$ & $149,54 \mathrm{c}$ \\
\hline Média & $2,10 \mathrm{~B}^{2}$ & $1,23 \mathrm{~A}$ & $1,12 \mathrm{~B}$ & $0,94 \mathrm{~A}$ & $336,04 \mathrm{~B}$ & $140,83 \mathrm{~A}$ \\
\hline $\mathrm{CV}(\%)$ & \multicolumn{2}{|c|}{7,13} & \multicolumn{2}{|c|}{8,75} & \multicolumn{2}{|c|}{7,63} \\
\hline
\end{tabular}

${ }^{1}$ Tratamentos estudados: T1 - testemunha, T2 - carbendazim + thiram na dose de 30,0 e 70,0g de i.a.100kg de sementes ${ }^{-1}$, respectivamente, $\mathrm{T} 3$ - fipronil + tiofanato metílico + piraclostrobina na dose de 50,0; 5,0 e 45,0g de i.a.100kg de sementes ${ }^{-1}$, respectivamente, e T4 abamectina + thiamethoxam + fludioxonil + mefenoxan+ thiabendazole, na dose de 62,$5 ; 70,0 ; 2,5 ; 1,0$ e $15,0 \mathrm{~g}$ de i.a. $100 \mathrm{~kg}^{\mathrm{de}} \mathrm{sementes}^{-1}$, respectivamente; ${ }^{2}$ Médias seguidas de mesma letra minúscula na coluna e maiúscula na linha, comparando os regimes hídricos (sem e com estresse hídrico) dentro de cada varíavel, não apresentam diferença significativa entre si pelo teste de Tukey ( $\mathrm{P}=0,05) ;{ }^{3} \mathrm{~S} . \mathrm{E} .:$ Sem estresse hídrico; ${ }^{4}$ C.E.: Com estresse hídrico.

produtividade da cultura (KRON et al., 2008). No entanto, as observações feitas neste ensaio indicaram que o tratamento de sementes limita a redução de área foliar induzida pelo déficit hídrico. Provavelmente, isso tenha acarretado aumento na taxa fotossintética, explicando os maiores valores de fitomassa verificados nesses tratamentos. Esse aumento também foi observado na ausência de déficit hídrico, porém, em menor magnitude.

Ensaio de campo

A análise de variância mostrou interação significativa entre os fatores regime hídrico e tratamentos de semente para todas as variáveis $(\mathrm{P} \leq 0,05)$. O estresse hídrico reduziu os valores da estatura de planta aos 28 e 42 dias após a emergência (Tabela 2) e rendimento de grãos.

O tratamento de sementes influenciou a emergência aos 7 e 28 dias após a emergência. Em ambas as avaliações, apenas os tratamentos com fipronil + tiofanato metílico + piraclostrobina (T3) e abamectina + thiamethoxam + fludioxonil + mefenoxan + thiabendazole (T4) promoveram incremento significativo na emergência de plantas, demonstrando que a combinação de inseticida/fungicida é vital para otimizar o benefício promovido pelo tratamento de sementes.

Em ambos os regimes hídricos, a diferença na estatura de planta aumentou no decorrer das avaliações, especialmente para os tratamentos com fipronil + tiofanato metílico + piraclostrobina (T3) e abamectina + thiamethoxam + fludioxonil + mefenoxan + thiabendazole (T4) comparados à testemunha. Sob baixa disponibilidade hídrica, houve diferença aos $14 \mathrm{e}$ 28 dias após a emergência, os tratamentos com fipronil + tiofanato metílico + piraclostrobina (T3) e abamectina + thiamethoxam + fludioxonil + mefenoxan + thiabendazole (T4) promoveram incremento na estatura das plantas. Sem estresse hídrico, os tratamentos com abamectina + thiamethoxam + fludioxonil + mefenoxan + thiabendazole (T4) foram superiores à testemunha nas avaliações aos 7 e 42 dias após a emergência.

Em ambos os regimes hídricos, o teor de clorofila foi maior nos tratamentos com fipronil + tiofanato metílico + piraclostrobina (T3) e abamectina + thiamethoxam + fludioxonil + mefenoxan + thiabendazole (T4). Essa constatação concorda com relatos no cultivo do trigo, em que a degradação de clorofila foi reduzida pela aplicação de kresoxim-methyl (GROSSMANN \& RETZLAFF, 1997). Esses resultados 
Tabela 2 - Efeito de diferentes tratamentos de sementes na evolução da estatura de plantas de soja (EP) (cm), teor de clorofila (SPAD), ataque de lagarta-elasmo (ALE) (\%), porcentagem de emergência de plantas (PE) (\%) e rendimento de grãos (RG) $\left(\mathrm{kg} \mathrm{ha}^{-1}\right)$ da cultivar de soja 'A 8100RG' na ausência e presença de estresse hídrico. Santa Maria, 2010.

\begin{tabular}{|c|c|c|c|c|c|c|c|c|c|c|}
\hline \multirow[t]{2}{*}{ Tratamentos $^{1}$} & \multicolumn{2}{|c|}{$7 \mathrm{DAE}^{5}$} & \multicolumn{2}{|c|}{ 14DAE } & \multicolumn{2}{|c|}{ 28DAE } & \multicolumn{2}{|c|}{ 42DAE } & \multicolumn{2}{|c|}{----ALE (\%)---- } \\
\hline & S.E $E^{3}$ & C.E. ${ }^{4}$ & S.E. & C.E. & S.E & C.E. & S.E & C.E. & S.E & C.E. \\
\hline $\mathrm{T} 1$ & $5,58 \mathrm{a}^{2}$ & $5,98 \mathrm{a}$ & $8,20 \mathrm{a}$ & $8,68 \mathrm{ab}$ & $23,35 \mathrm{a}$ & $21,21 \mathrm{ab}$ & $42,22 \mathrm{a}$ & $39,60 \mathrm{a}$ & - & $37,41 \mathrm{c}$ \\
\hline $\mathrm{T} 2$ & $5,76 \mathrm{ab}$ & $5,75 \mathrm{a}$ & $8,51 \mathrm{a}$ & $8,09 \mathrm{a}$ & $23,40 \mathrm{a}$ & $19,70 \mathrm{a}$ & $44,27 \mathrm{ab}$ & $40,30 \mathrm{a}$ & - & $38,04 \mathrm{c}$ \\
\hline $\mathrm{T} 3$ & $5,95 \mathrm{ab}$ & $6,00 \mathrm{a}$ & $8,63 \mathrm{a}$ & $8,75 \mathrm{~b}$ & $26,41 \mathrm{a}$ & $21,26 \mathrm{ab}$ & $50,97 \mathrm{bc}$ & $44,82 \mathrm{a}$ & - & $7,22 \mathrm{a}$ \\
\hline $\mathrm{T} 4$ & $6,28 \mathrm{~b}$ & $6,25 \mathrm{a}$ & $8,61 \mathrm{a}$ & $8,35 \mathrm{ab}$ & $25,81 \mathrm{a}$ & $21,94 \mathrm{~b}$ & $52,25 \mathrm{c}$ & $44,65 \mathrm{a}$ & - & $19,55 \mathrm{~b}$ \\
\hline Média & $5,89 \mathrm{~A}^{2}$ & $6,00 \mathrm{~A}$ & $8,48 \mathrm{~A}$ & $8,46 \mathrm{~A}$ & $24,74 \mathrm{~B}$ & $21,03 \mathrm{~A}$ & 47,43 B & $42,34 \mathrm{~A}$ & - & 25,56 \\
\hline $\mathrm{CV}(\%)$ & \multicolumn{2}{|c|}{5,45} & \multicolumn{2}{|c|}{4,57} & \multicolumn{2}{|c|}{5,33} & \multicolumn{2}{|c|}{6,93} & - & 20,40 \\
\hline \multirow{2}{*}{ Tratamentos $^{1}$} & \multicolumn{2}{|c|}{--------SPAD-------- } & \multicolumn{4}{|c|}{ 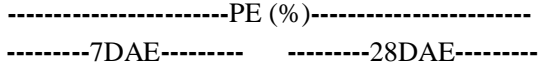 } & \multicolumn{4}{|c|}{ 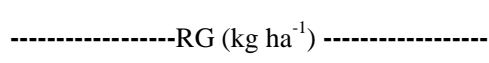 } \\
\hline & S.E $E^{3}$ & C.E. ${ }^{4}$ & S.E & C.E. & S.E & C.E. & \multicolumn{2}{|c|}{ S.E } & \multicolumn{2}{|c|}{ C.E. } \\
\hline $\mathrm{T} 1$ & $28,58 \mathrm{a}^{2}$ & $28,65 \mathrm{a}$ & $65,06 \mathrm{a}$ & $62,05 \mathrm{a}$ & $57,83 \mathrm{a}$ & $52,41 \mathrm{a}$ & \multicolumn{2}{|c|}{$2917,58 \mathrm{a}$} & \multicolumn{2}{|c|}{$1449,68 \mathrm{a}$} \\
\hline $\mathrm{T} 2$ & $29,93 \mathrm{ab}$ & 29,48 a & $67,62 \mathrm{a}$ & $72,74 \mathrm{ab}$ & $62,95 \mathrm{ab}$ & $57,83 a b$ & \multicolumn{2}{|c|}{3014,80 a } & \multicolumn{2}{|c|}{$1612,68 \mathrm{ab}$} \\
\hline $\mathrm{T} 3$ & $31,75 \mathrm{~b}$ & $33,32 \mathrm{~b}$ & $83,88 \mathrm{~b}$ & $75,90 \mathrm{ab}$ & $76,05 \mathrm{ab}$ & $64,61 \mathrm{ab}$ & \multicolumn{2}{|c|}{2918,70 a } & \multicolumn{2}{|c|}{$2287,13 \mathrm{~b}$} \\
\hline $\mathrm{T} 4$ & $31,42 \mathrm{~b}$ & $32,54 \mathrm{~b}$ & $89,60 \mathrm{~b}$ & $80,87 \mathrm{~b}$ & $83,58 \mathrm{~b}$ & $72,44 \mathrm{~b}$ & \multicolumn{2}{|c|}{$2754,54 \mathrm{a}$} & \multicolumn{2}{|c|}{$2282,22 \mathrm{~b}$} \\
\hline Média & $30,42 \mathrm{~A}^{2}$ & $31,00 \mathrm{~A}$ & $76,54 \mathrm{~A}$ & $72,89 \mathrm{~A}$ & $70,10 \mathrm{~A}$ & $61,82 \mathrm{~A}$ & \multicolumn{2}{|c|}{2950,36 B } & \multicolumn{2}{|c|}{$1907,93 \mathrm{~A}$} \\
\hline $\mathrm{CV}(\%)$ & \multicolumn{2}{|c|}{3,37} & \multicolumn{2}{|c|}{9,40} & \multicolumn{2}{|c|}{13,35} & \multicolumn{4}{|c|}{13,14} \\
\hline
\end{tabular}

${ }^{1}$ Tratamentos estudados: T1 - testemunha, T2 - carbendazim + thiram na dose de 30,0 e 70,0g de i.a.100kg de sementes ${ }^{-1}$, respectivamente, $\mathrm{T} 3$ - fipronil + tiofanato metílico + piraclostrobina na dose de 50,0;5,0 e 45,0g de i.a.100kg de sementes ${ }^{-1}$, respectivamente, e T4 abamectina + thiamethoxam + fludioxonil + mefenoxan+ thiabendazole, na dose de 62,5;70,0;2,5;1,0 e 15,0g de i.a.100kg de sementes ${ }^{-1}$, respectivamente; ${ }^{2}$ Médias seguidas de mesma letra minúscula na coluna e maiúscula na linha, comparando os regimes hídricos (sem e com estresse hídrico) dentro de cada variável, não apresentam diferença significativa entre si pelo teste de Tukey $(\mathrm{P}=0,05) ;{ }^{3}$ S.E.: Sem estresse hídrico; ${ }^{4}$ C.E.: Com estresse hídrico; ${ }^{5}$ DAE: Dias após a emergência das plantas.

podem ser explicados devido às estrobilurinas inibirem alguns precursores da síntese de etileno como a ACC sintase e ACC oxidase. O etileno é um hormônio que está envolvido na degradação da clorofila (TAIZ \& ZEIGER, 2009) e a redução na atividade dessas enzimas pode atrasar a degradação da clorofila (GOLD \& LEINHOS, 1995; GROSSMANN \& RETZLAFF, 1997). Em soja, o thiamethoxam promove a expressão de genes relacionados com a síntese e atividade de enzimas, alterando a produção de aminoácidos precursores de fitohormônios, o que resulta em aumento de germinação, vigor e comprimento radicular (CASTRO et al., 2008).

$\mathrm{O}$ ataque de lagarta-elasmo foi observado no regime com restrição hídrico, acarretando redução na população devido tombamento de plantas. $\mathrm{Na}$ parcela testemunha (T1) e no tratamento sem inseticida (T2), o percentual de plantas atacadas atingiu $37,41 \%$ e $38,04 \%$, respectivamente. Ambos os tratamentos que continham inseticida reduziram a incidência da praga. O tratamento com fipronil + tiofanato metílico + piraclostrobina (T3) foi o mais eficiente, reduzindo em
$81 \%$ o número de plantas atacadas em relação à testemunha.

O rendimento de grãos não foi influenciado pelos tratamentos de semente no regime sem estresse hídrico. Na presença de estresse hídrico, no entanto, houve diferença entre eles. Nesse caso, o maior rendimento de grãos foi obtido nos tratamentos com fipronil + tiofanato metílico + piraclostrobina (T3) e abamectina + thiamethoxam + fludioxonil + mefenoxan + thiabendazole (T4), sendo 57,8\% e 57,4\% superior à testemunha, respectivamente.

Plantas de soja que receberam a aplicação de piraclostrobina, na ausência de doenças foliares, apresentaram aumento de $10 \%$ na massa seca, mas não ocorreu incremento na produtividade (SWOBODA \& PEDERSEN, 2009). Esses dados corroboram com os resultados deste estudo, no qual, na ausência de estresse hídrico, não foi observado incremento significativo no rendimento de grãos pela aplicação da molécula na semente. Na presença de estresse hídrico, não foram encontrados dados que relacionem a aplicação de estrobilurinas e rendimento de grãos. 
O incremento no rendimento de grãos foi observado somente na presença de estresse hídrico nas sementes tratadas com fipronil + tiofanato metílico + piraclostrobina (T3). Isso pode ser explicado devido ao controle da lagarta-elasmo, bem como por alterações fisiológicas que aumentam a tolerância das plantas ao estresse, possivelmente devido ao aumento nos níveis endógenos de ácido abscísico. Discos foliares de trigo submetidos ao tratamento com kresoxim-methyl apresentaram aumento de duas vezes nos níveis endógenos de ácido abscísico (GROSSMANN et al., 1999). Concomitantemente, a abertura estomatal e o consumo de água foram reduzidos. Em contrapartida, a eficiência de uso da água (EUA) de plantas de soja, trigo e cevada pela aplicação de piraclostrobina, azoxistrobina e trifloxistrobina foi melhorada somente em condições de boa disponibilidade hídrica (NASON et al., 2007). Sob déficit hídrico, a EUA foi reduzida comparada à testemunha. Nesse caso, a taxa de transpiração, a concentração intercelular de $\mathrm{CO}_{2}$ e a taxa fotossintética foram menores nos tratamentos com estrobilurinas. Os autores citam que esse efeito pode ser devido à inibição da produção de ATP pela célulaguarda do estômato. A produção reduzida de ATP pode prejudicar o metabolismo da célula-guarda, diminuindo a pressão de turgor e, por conseguinte, a concentração de $\mathrm{CO}_{2}$ e a taxa fotossintética (NASON et al., 2007).

Os resultados obtidos a partir do conjunto de ensaios relatados demonstraram que o tratamento de sementes com compostos que contém thiamethoxam e piraclostrobina se constitui em uma alternativa bastante viável para a melhoria de alguns parâmetros fisiológicos da cultura da soja. Conclui-se que o tratamento de sementes com esses produtos confere alterações benéficas na planta, aumentando sua tolerância ao estresse hídrico, com efeito positivo sobre o rendimento de grãos da cultura da soja. Estudos avaliando a concentração de alguns hormônios, como o etileno e ácido abscísico em resposta ao tratamento de sementes em condições de déficit hídrico, serão de suma importância para elucidar os mecanismos bioquímicos envolvidos no aumento de tolerância induzido por esses compostos.

\section{REFERÊNCIAS}

BAUDET, L.; PESKE, F. Aumentando o desempenho das sementes. Seed News, v.9, n.5, p.22-24, 2007. Dísponivel em: <http:// www.seednews.inf.br/portugues/seed115/print_artigo115.html>. Acesso em: 02 jul. 2010.

CASTRO, G.S.A. et al. Tratamento de sementes de soja com inseticidas e um bioestimulante. Pesquisa Agropecuária Brasileira, v.43, n.10, p.1311-1318, 2008. Dísponivel em: <http://www.scielo.br/pdf/pab/v43n10/08.pdf>. Acesso em: 25 jul. 2010. doi 10.1590/S0100-204X2008001000008.
COMISSÃO DE QUíMICA E FERTILIDADE DO SOLO. Manual de adubação e calagem para os estados do Rio Grande do Sul e Santa Catarina. 10.ed. Porto Alegre: Sociedade Brasileira de Ciência do Solo, 2004. 394p.

CORNIC, G. Drought stress inhibits photosynthesis by decreasing stomatal aperture - not by affecting ATP synthesis. Trends in Plant Science, London, v.5, p.183-221, 2000. Dísponivel em: $\langle$ http://dx.doi.org/10.1016/S1360-1385(00)01625-3>. Acesso em: 29 jul. 2010 . doi: 10.1016/S1360-1385(00)01625-3.

COSTAMILAN, L.M. et al. La Niña e os possíveis efeitos sobre a ocorrência de doenças de soja na safra 2010/ 2011. Disponível em: <http://www.cnpt.embrapa.br/pesquisa/ fitopatologia/LaNina_ocorrencia_doencas_soja20102011.pdf>. Online. Acesso em: 05 dez. 2010.

GOLD, R.E.; LEINHOS, G. M. Fungicidal effects of BAS 490F on the development and fine structure of plant pathogenic fungi. Pesticide Science, v.43, p.250-253, 1995. Dísponivel em: <http://dx.doi.org/10.1002/ps.2780430316>. Acesso em: 02 set. 2010 . doi: $10.1002 /$ ps.2780430316.

GROSSMANN, K. et al. Regulation of phytohormone levels, leaf senescence and transpiration by the strobilurin kresoximmethyl in wheat (Triticum aestivum). Journal of Plant Physiology, v.154, n.5-6, p.805-808, 1999. Dísponivel em: <http://www.seedquest.com/id/b/basf/vitality/articles/ Regulation_of_phytohormone_levels.pdf $>$. Acesso em: 04 set. 2010 .

GROSSMANN, K.; RETZLAFF, G. Bioregulatory effects of the fungicidal strobilurin kresoxim-methyl in wheat (Triticum aestivum). Pesticide Science, v.50, p.11-20, 1997. Disponivel em: <http://seedquest.securesites.net/id/b/basf/ vitality/articles/Strobilurin_Kresoximmethyl_in_Wheat.pdf $>$. Acesso em 04 set. 2010.

KRON, A.P. et al. Water deficiency at different developmental stages of Glycine max can improve drought tolerance. Bragantia, v.67, n.1, 43-49, 2008. Dísponivel em: <http:// www.scielo.br/pdf/brag/v67n1/a05v67n1.pdf >. Acesso em: 30 jul. 2010. doi: 10.1590/S0006-87052008000100005.

NASON, M.A. et al. Strobilurin fungicides induce changes in photosynthetic gas exchange that do not improve water use efficiency of plants grown under conditions of water stress. Pest Managagement Science, v.63, p.1191-1200, 2007. Dísponivel em: < http://dx.doi.org/10.1002/ps.1443>. Acesso em: 02 set. 2010 . doi: 10.1002/ps. 1443 .

PEREIRA, L.A.G. et al. Tratamento de sementes de soja com fungicida e/ou antibiótico, sob condições de semeadura em solo com baixa disponibilidade hídrica. Revista Brasileira de Sementes, v.15, n.2, p.241-246, 1993. Dísponivel em: <http:/ /www.scielo.br/pdf/cr/v39n1/a03v39n1.pdf>. Acesso em: 30 de jul. 2010. doi: 10.1590/S0103-84782009000100003.

SANTOS, M.G. et al. The role of inorganic phosphate on photosynthesis recovery of common bean after a mild drought deficit. Plant Science, Clare, v.170, p.659-664, 2006. Dísponivel em: <http://dx.doi.org/10.1016/j.plantsci.2005.10.020〉. Acesso em: 07 set. 2010. doi: 10.1016/j.plantsci.2005.10.020. 
SANTOS, M.G. et al. Gas exchange and yield response to foliar phosphorus application in Phaseolus vulgaris L. under drought. Brazilian Journal of Plant Physiology, v.16, p.171-179, 2004. Dísponivel em: <http://www.scielo.br/pdf/bjpp/v16n3/ 22854.pdf >. Acesso em: 07 set. 2010. doi: 10.1590/S167704202004000300007 .

SWOBODA, C.; PEDERSEN, P. Effect of fungicide on soybean growth and yield. Agronomy Journal, v.101, p.352-356, 2009. Dísponivel em: 〈http://dx.doi.org/10.2134/agronj2008.0150〉. Acesso em: 03 set. 2010. doi: 10.2134/agronj2008.0150.
TAIZ, L; ZEIGER, E. Fisiologia vegetal. Tradução: SANTARÉM, E.R. [et al.]. 4.ed. Porto Alegre: Artmed, 2009. $848 \mathrm{p}$.

TAVARES, S. et al. Avaliação dos efeitos fisiológicos de thiametoxan no tratamento de sementes de soja. Revista de Agricultura, v.82, p.47-54, 2007.

Vale, F.X.R. Do; Fernandes Filho, E.I.; Liberato, J.R.; Zambolim, L.. Quant a software to quantify plant disease severity In: International workshop on plant disease epidemiology, 8., 2001, Ouro Preto. Ouro Preto: International Society of Plant Pathology, 2001. 160p. 